\title{
Novel Quinolizine AIE System: Visualization of Molecular Motion and Elaborate Tailoring for Biological Application
}

Benzhao He, Jiachang Huang,* Jianyu Zhang, Herman H. Y. Sung, Jacky W. Y. Lam, Zhijun Zhang, Saisai Yan, Dong Wang, * Jing Zhang, * and Ben Zhong Tang*

Dr. B. He, Dr. J. Huang

Center for Advanced Materials Research

Science and Technology Experimental Platform

Advanced Institute of Natural Sciences

Beijing Normal University at Zhuhai, Zhuhai, 519085, China

Email: jiachang_huang9021@163.com

Mr. J. Zhang, Dr. H. H. Y. Sung, Dr. J. W. Y. Lam, Prof. B. Z. Tang

Department of Chemistry

Hong Kong Branch of Chinese National Engineering Research Center for Tissue Restoration and Reconstruction

The Hong Kong University of Science \& Technology, Clear Water Bay, Kowloon, Hong Kong, China

Email: tangbenz@cuhk.edu.cn

Dr. J. W. Y. Lam, Prof. B. Z. Tang

HKUST-Shenzhen Research Institute,

No. 9 Yuexing 1st RD, South Area, Hi-tech Park, Nanshan, Shenzhen, 518057, China

Prof. D. Wang, Dr. Z. Zhang, Dr. S. Yan

Center for AIE Research

College of Materials Science and Engineering

Shenzhen University

Shenzhen 518060, China,

Email: wangd@szu.edu.cn

Prof. J. Zhang

Department of Laboratory Medicine

Nanfang Hospital, Southern Medical University

Guangzhou 510515, China

Email: zjsilence@i.smu.edu.cn

Prof. B. Z. Tang

Shenzhen Institute of Aggregate Science and Technology, School of Science and Engineering, The Chinese University of Hong Kong, Shenzhen, 2001 Longxiang Boulevard, Longgang District, Shenzhen, 518172, China, Email: tangbenz@cuhk.edu.cn 


\begin{abstract}
:
Molecular motions are ubiquitous in nature and they immutably play intrinsic roles in all actions. However, exploring appropriate models to decipher molecular motions is an extremely important but very challenging task for researchers. Considering aggregation-induced emission (AIE) luminogens possess their unique merits to visualize molecular motions, it is particularly fascinating to construct new AIE systems as model to study molecular motion. Herein, a novel quinolizine (QLZ) AIE system was constructed based on the restriction intramolecular vibration mechanism. It was demonstrated that QLZ could act as an ideal model to visualize single-molecule motion and macroscopic molecular motion via fluorescence change. Additionally, further elaborate tailoring of this impressive core achieved highly efficient reactive oxygen species production and realized fluorescence imaging-guided photodynamic therapy applications, which confirms the great application potential of this new AIE-active QLZ core. Therefore, this work not only provides an ideal model to visualize molecular motion but also opens a new way for the application of AIEgens.
\end{abstract}




\section{Introduction}

Molecular motions are ubiquitous in nature. From microscopic level to mesoscopic level and to macroscopic level, molecular motions immutably play their intrinsic role in all actions. ${ }^{[1]}$ Accordingly, the decipher of molecular motions in different levels has been a significant research subject all the time, ${ }^{[2]}$ which would provide important guidance for researchers to manipulate and make full use of them to achieve unprecedented creation. It is noteworthy that visualization is the most direct and reliable way to study molecular motions that can directly represent the multifarious states of molecular motions. As an ideal kind of visualization model, aggregation-induced emission luminogens (AIEgens) recently have demonstrated their unprecedented advantages in the exploration of molecular motions. ${ }^{[2 b, 3]}$

After continuous efforts, to date, it has been well elucidated and widely accepted that the emission mechanism of AIE is restriction of intramolecular motion (RIM), including two aspects, namely, the restriction of intramolecular rotation (RIR) and the restriction of intramolecular vibration (RIV). ${ }^{[4]}$ Specifically, when the molecules are separately dispersed in solution, the active intramolecular motion will facilitate the non-radiative decay channels and cause non-emissive state; while once forming aggregates, the non-radiative decay channels will be blocked due to the RIM resulting in enhanced emission. In this aspect, AIEgens possess their unique merits to visualize molecular motion. ${ }^{[4 b]}$ In recent years, numerous new AIE systems have been developed to achieve various kinds of applications from materials science to biotechnology and pharmacotherapy. ${ }^{[5]}$ It can be concluded that the majority of organic AIE systems are constructed from RIR mechanism, especially those based on some classical cores,

such as aryl ethylene, triphenylamine (TPA), hexaphenylsilole (HPS), etc. ${ }^{[6]}$ However, the developed RIV-based AIE systems are relatively less since the discovery of 10,10',11,11'- 
tetrahydro-5,5'-bidibenzo[a,d][7] annulenylidene

bidibenzo[a,d][7]annulenylidene (BDBA)..$^{[4 a, 6-7]}$ Considering the RIV systems are also desirable and indispensable models to study molecular motion, it is highly in demand to construct new RIV-based AIE core. More importantly, the development of novel AIE cores can provide more extensive space for the derivation of new materials and further broaden the application scope of AIEgens.

It should be mentioned that quinolizines are a class of important compounds that have similar structures to isoquinoline and they are widely existed in numerous biologically active alkaloids. ${ }^{[8]}$ As early as 1932, Diels and Alder have reported quinolizine compounds that were constructed by a simple 1,4-dipolar cycloaddition reaction based on dimethyl acetylenedicarboxylate and pyridine. ${ }^{[9]}$ Accordingly, the preparation of quinolizines is very facile and efficient given that the raw materials are the cheap, versatile and easily available pyridines. ${ }^{[10]}$ It has been well documented that quinolizine rings play important roles in many antihypertensive, antibacterial, and anticancer drugs. ${ }^{[11]}$ However, to the best of our knowledge, there were no any study to present their specific structures and report their luminescent properties so far. We are highly interested in this special structure considering that it is an 8electron conjugated structure and should display nonaromatic character, where the two rings are probably non-coplanar. Since the non-coplanarity of the structure may lead to intramolecular vibration to quench its emission, the suppression of which theoretically can boost the emission. So we presume that quinolizine should be a unique AIE system featuring RIV that can act as an ideal model to investigate molecular motion.

Inspired by the above, we herein attempted to synthesize the tetramethyl $4 H$-quinolizine- 
1,2,3,4-tetracarboxylate (QLZ) molecule by a 1,4-dipolar cycloaddition reaction based on dimethyl acetylenedicarboxylate and pyridine, and further attained its accurate structure for the first time. As envisaged, the two rings of QLZ are indeed non-coplanar (see below for structural details) implying its inherent molecular vibration. After systematic and comprehensive exploration, it turns out that QLZ possesses impressive AIE property via RIV and it could act as an ideal model to visualize single-molecule motion and macroscopic molecular motion. As a novel RIV-based AIE core, we further elaborately tailored its structure to demonstrate its potential in biological application and ultimately realized the bioimaging and efficient photodynamic therapy (PDT) of cancer. Therefore, this work provides new insights into the exploration of molecular motion and the developed new AIE core will open a new way for the application of AIEgens.

\section{Results and Discussion}

\section{Molecular Synthesis and Photophysical Properties}

The target compound QLZ was prepared by simple one-step 1,4-dipolar cycloaddition reaction of dimethyl acetylenedicarboxylate (1) with pyridine (2) under mild conditions (Figure 1A, see the Supporting Information for details). The structure of QLZ was characterized using nuclear magnetic resonance (NMR) and high-resolution mass spectroscopy (HRMS) with satisfactory results (Figures S1-S3). Additionally, the single crystal of QLZ was successfully obtained by solvent evaporation, and the molecular structure was further confirmed by singlecrystal X-ray diffraction (details see below). The relevant data were provided in Table S1. As our previous conjecture, the two rings of the obtained multi-substituted $4 H$-quinolizine structure are indeed non-coplanar and the entire molecular skeleton is bent (Figure 1A), which 
is considered to provide sufficient conditions for the intramolecular vibrations.

After successful construction, its photophysical properties were further systematically investigated. As showed in Figure S4, QLZ exhibited a strong absorption band at $450 \mathrm{~nm}$. When excited with $450 \mathrm{~nm}$ at room temperature, its dilute tetrahydrofuran (THF) solution exhibited an orange-red emission at $610 \mathrm{~nm}$ (Figure 1B). However, when the temperature was cooled to $77 \mathrm{~K}$, the emission wavelength showed a prominent blueshift $(\sim 80 \mathrm{~nm})$ and the corresponding intensity exhibited a sharp enhancement (Figure 1B). Based on this remarkable change and combining its unique bent structure, we presumed that the structure of QLZ after excitation should undergo a bent-to-planar configuration transition at room temperature, which leads to a non-radiative attenuation and the decrease of the energy gap (Figure S5), so it ultimately exhibited weak and orange-red fluorescence; while at low temperature, the structural relaxation toward planarization would be impeded and the non-radiative transition should be suppressed, thus the luminescence blue shifts and enhances. ${ }^{[12]}$ If so, the luminescent change of QLZ in turn perfectly visualized its molecular vibration. It has been well documented that aggregation is a good way to regulate molecular motion. ${ }^{[13]}$ To further check whether QLZ possesses the property of AIE, we study its emission behavior of QLZ in different aggregation states. As shown in Figure 1C, the diluted THF solution of QLZ exhibited week emission. When a poor solvent of water was added, the emission intensity gradually increased and the strongest intensity was reached at water fraction $\left(f_{\mathrm{w}}\right)$ of $80 \%$, which was a 3.7 -fold higher than that in pure THF solution (Figure 1D). These results suggest its typical AIE performance. ${ }^{[14]}$ Taken together, these results further indicate that the constructed quinolizine ring could serve as a novel AIE core. It should be noted that the emission intensity of QLZ was enhanced in the 
aggregate state indicating that molecular aggregate induced the restriction of intramolecular motion to a certain extent, but its emission wavelength showed no obvious change revealing that the formed aggregates are relatively loose where the bent-to-planar intramolecular motion could still occur.

A
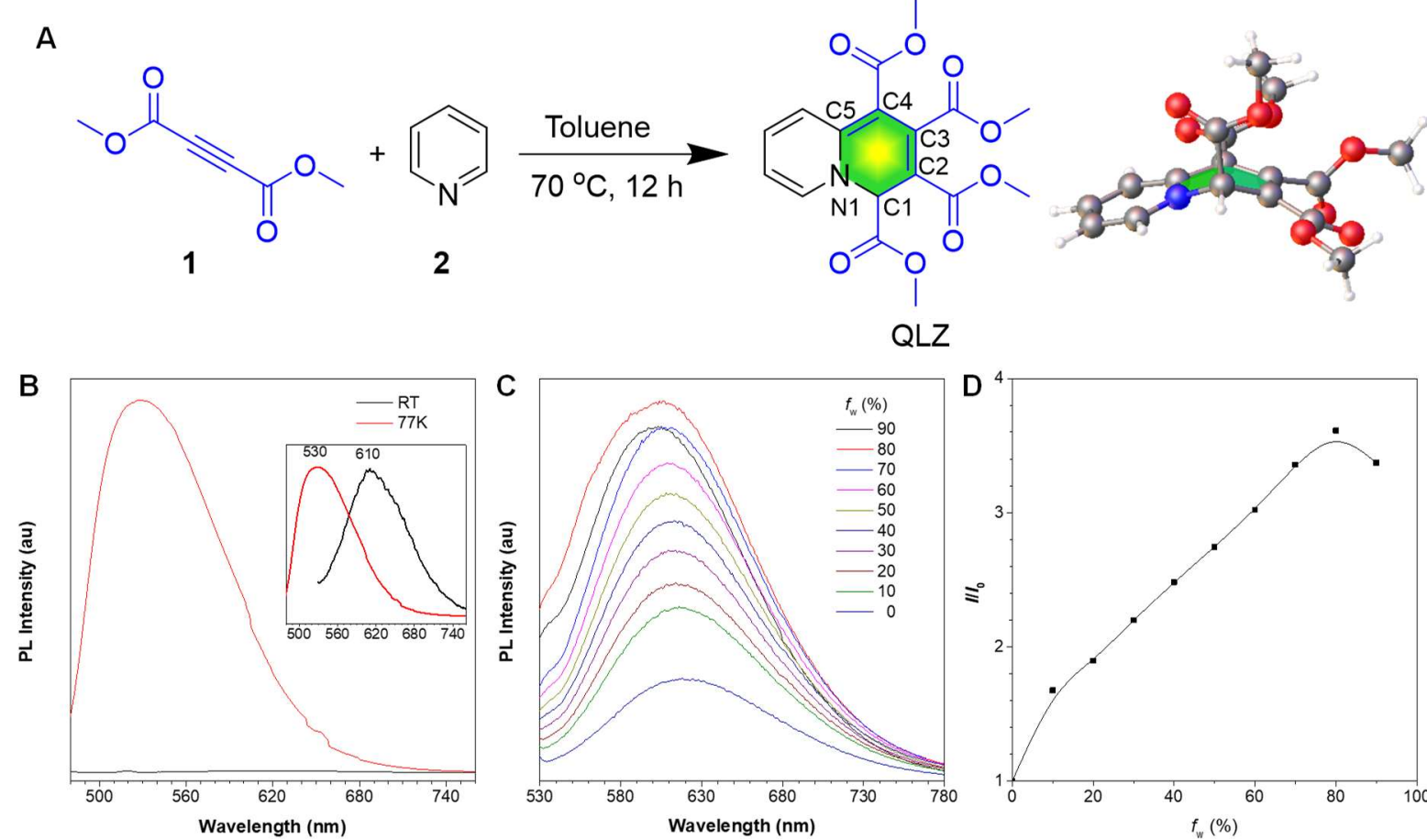

Figure 1. (A) The synthetic route of QLZ and its corresponding single crystal structure (CCDC: 2114005). (B) Photoluminescence (PL) spectra of QLZ in THF at room temperature and 77K, respectively $(10 \mu \mathrm{M})$. (C) PL spectra of QLZ in THF/water mixtures with different water fractions $\left(f_{\mathrm{w}}\right)$. (D) Plot of relative emission intensity $\left(I / I_{0}\right)$ versus the water fraction of the aqueous mixtures of QLZ $\left(\lambda_{\mathrm{ex}}=450 \mathrm{~nm}, 10 \mu \mathrm{M}\right)$.

\section{Theoretical revelation of molecular motion}

In order to better understand the molecular motion-associated luminescence mechanism, density functional theory (DFT) calculations based on QLZ were carried out. According to the calculated results in Figure 2A, the quinolizine ring forms a non-planar structure, where the dihedral angle between the two planes formed by $\mathrm{C} 1-\mathrm{C} 2-\mathrm{C} 3$ and $\mathrm{C} 1-\mathrm{N} 1-\mathrm{C} 5$ is $145.94^{\circ}$ in the ground state. Comparatively, this dihedral angle becomes $158.99^{\circ}$ in the excited state, which proves our hypothesis mentioned above that the QLZ molecule can undergo a bent-to-planar 
geometrical change after excitation. Furthermore, it is also confirmed that the AIE characteristic of QLZ is attributed to RIV. In addition, the calculated absorption peak and emission peak of QLZ are located at $418 \mathrm{~nm}$ and $635 \mathrm{~nm}$, respectively (Figure 2A and Figure S5), which are well consistent with the experimental results.

It has been well accepted that reorganization energy $(\lambda)$ can quantitatively indicate the intrinsic geometric change upon photoexcitation, and it can directly present the contribution of different types of intramolecular motion to nonradiative annihilate. ${ }^{[15]}$ The calculated $\lambda$ of QLZ at different wavenumbers in the isolated solution phase was displayed in Figure 2B. We can see that the total $\lambda$ was $5435 \mathrm{~cm}^{-1}$ where $62.41 \%$ makes contribution to the change of dihedral angle that was attributed to the vibration of quinolizine rings. Several motion modes of QLZ based on optimized excited state geometry were depicted in Figure S6, which well demonstrated the intramolecular vibration in the low-frequency region. It should be mentioned that these low-frequency vibration modes tended to mix when excited to activate multiple nonradiative decay pathways. ${ }^{[16]}$ Comparatively, according to the results of QM/MM calculation in the crystalline phase (Figure S7), it was found that the total $\lambda$ decreased to 4063 $\mathrm{cm}^{-1}$, where the contribution of the dihedral angle declined to $11.72 \%$, while the contribution of the bond length increased to $75.16 \%$ (Figure 2C). These obvious changes reinforce the restriction of intramolecular vibration of QLZ in the excited state, further proving that the emission of QLZ is caused by molecular vibrations. 

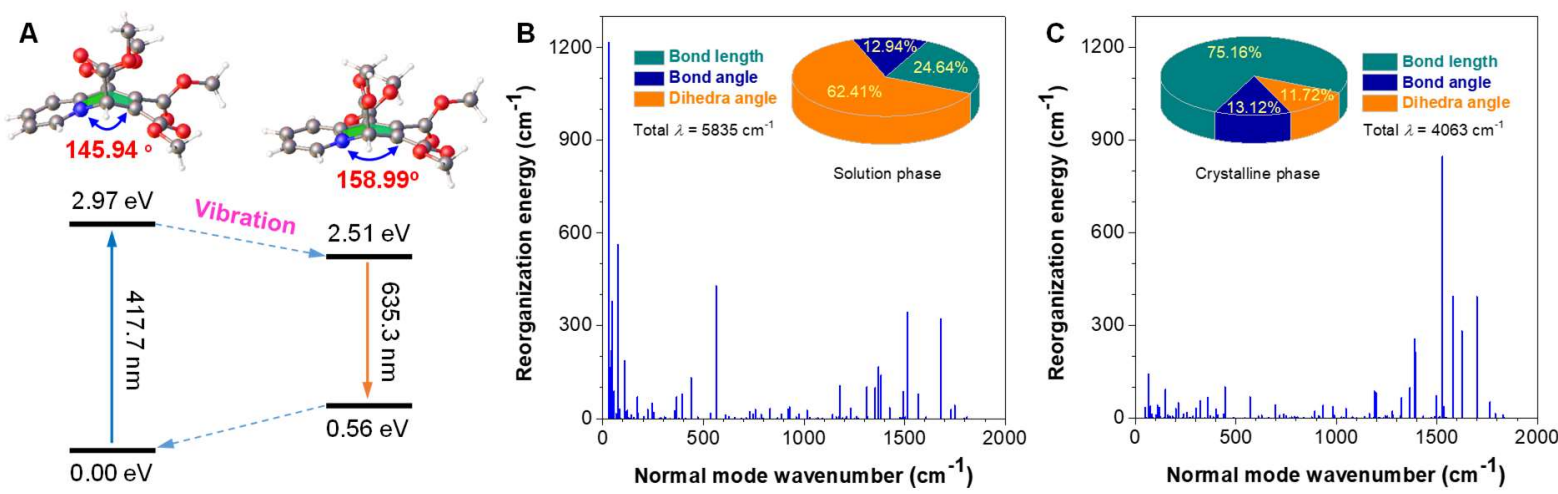

Figure 2. (A) Illustrative scheme for VIE involved excited-state configuration transformation from bent to planar. Plots of calculated reorganization energy versus the normal mode wavenumber of QLZ in the isolated solution phase (B) and crystalline phase (C), calculated by the TD-DFT method at B3LYP/6-31G $(\mathrm{d}, \mathrm{p})$ level of Gaussian 16 program and MOMAP.

\section{Visualization of Solid-State Molecular Motion}

Based on the above results, we have noted impressive molecular motions for QLZ in the solution state. Regarding its solid state, we also conducted detailed investigation. Interestingly, we observed two different colors of luminescence for the crystal of QLZ when growing its single crystals, which implies that QLZ may have varied emission. This phenomenon aroused our great interest to study its crystals. As demonstrated in Figure 3A-3D and Video in Supporting Information, the inner part of the pristine crystal emits green light, while the surface of the crystal emits orange-red emission. Surprisingly, when we lightly ground the large crystals into small crystals, the resulting crystals exhibited much brighter green emission, and the corresponding quantum yield (QY) increased to 8.5\% from the original 3.3\%. Further heavily grinding, it was observed that the emission of small crystal turned into weak orangered. After completely ground heavily, the emission of all the samples became orange-red, and QY drop to $2.8 \%$. The PL spectral of QLZ in different states were summarized in Figure S8.

In order to unveil these interesting phenomena, we employed powder X-ray diffraction (PXRD) to characterize the crystalline changes of QLZ in different states, and the results were 
presented in Figure 3E. We can see that the pristine crystal of QLZ exhibited strong diffraction peaks, which were well consistent with those simulated from the single crystal X-ray data. It was surprised that the patterns of PXRD showed no change for the sample after slight grinding, which indicates that there was no any change for the crystal phase after slight grinding. But we observed so obvious emission change during the gently grinding process. Taken these information together and combining our experience, ${ }^{[2 \mathrm{~b}]}$ we reasoned that the influence of slight grinding on molecular motions was relatively subtle and did not cause the transformation of crystal phase, but luminescence was so sensitive that it could signal this subtle change. However, after ground heavily, the diffraction peaks broadened and weakened, demonstrating the crystal phase have transformed into amorphous state from crystalline state. This obvious change indicates that heavy grinding should result in the occurrence of noteworthy molecular motions.
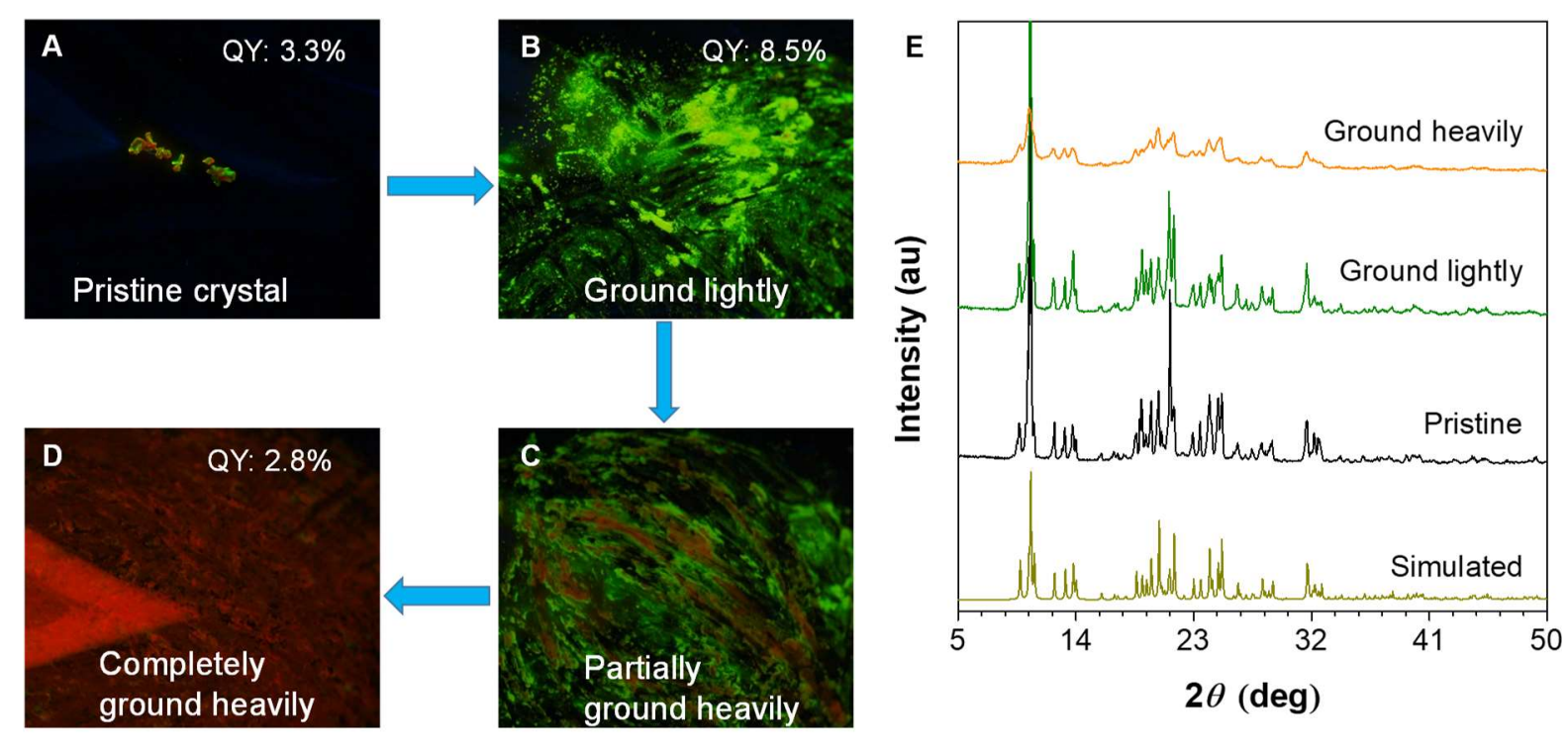

Figure 3. Pictures of QLZ in different solid states taken under UV irradiation at $365 \mathrm{~nm}$. (A) Pristine crystal, (B) ground lightly, (C) partially ground heavily, (D) completely ground heavily. (E) Powder X-ray diffraction patterns of QLZ in different states.

Fortunately, single crystal structure of QLZ with dual emission was obtained, which can 
provide more detailed information for us to study the above phenomena. We can clearly observe that the surface of the pristine crystal emits orange-red luminescence, while the inner part exhibited green emission (Figure 3A). Here, we should point out that we have tried many times to obtain the crystals with single-color luminescence, but failed. This may in turn imply that the state with dual luminescence is relatively stable. As illustrated in Figure 4A, there are multiple strong hydrogen bonds between the neighboring QLZ molecules and these strong interactions drive the molecules to form tight layer-by-layer packing (Figure 4A and Figure S9). When we carefully studied the intermolecular packing of QLZ crystal, we further found that the inner molecules of the packing are arranged very tightly, while the outer molecules of in yellow the crystal are arranged very loosely and their number are relatively less (Figure 4A and Figure S9), which provides important hint for the dual luminescence of QLZ crystal. Herein, we can reason that the loose packing renders the outer molecules to have enough space to undergo a bent-to-planar configuration transition upon excitation, thus the surface of the crystal emits orange-red luminescence. While the tight packing restricts the intramolecular vibration of the inner molecules, thus the inner part of the crystal emits the inherent green fluorescence. Until now, combining all the experimental results with the present crystal data as well as the calculated results, we can propose a reasonable mechanism for the impressive mechanical force-induce luminescent changes. ${ }^{[17]}$ Specifically, in the pristine large crystal, the molecules on the surface arrange loosely and the intermolecular interaction are relatively weak. When we gently ground the large crystals into small crystals, the slight mechanical force is enough to influence them and induces the outer molecules to rearrange more closely, which restricts the original molecular vibrations and results in green luminescence identical to the 
inner part. This subtle change does not induce the transformation of crystal state, but luminescence is sensitive enough to visualize this subtle change. Regarding the further heavy grinding process, the strong mechanical force doubtlessly can destroy all the stacking of QLZ crystals and induce a macroscopical alteration of the crystal phase from crystal state to amorphous state. The resulting disordered molecules recover their free intramolecular vibrations, thus QLZ ultimately emits orange-red luminescence similar to its solution state at room temperature. All in all, this new AIE core has provided us a useful tool to visualize both the subtle single molecular motion and the macroscopic molecular motions via the super sensitive luminescence.

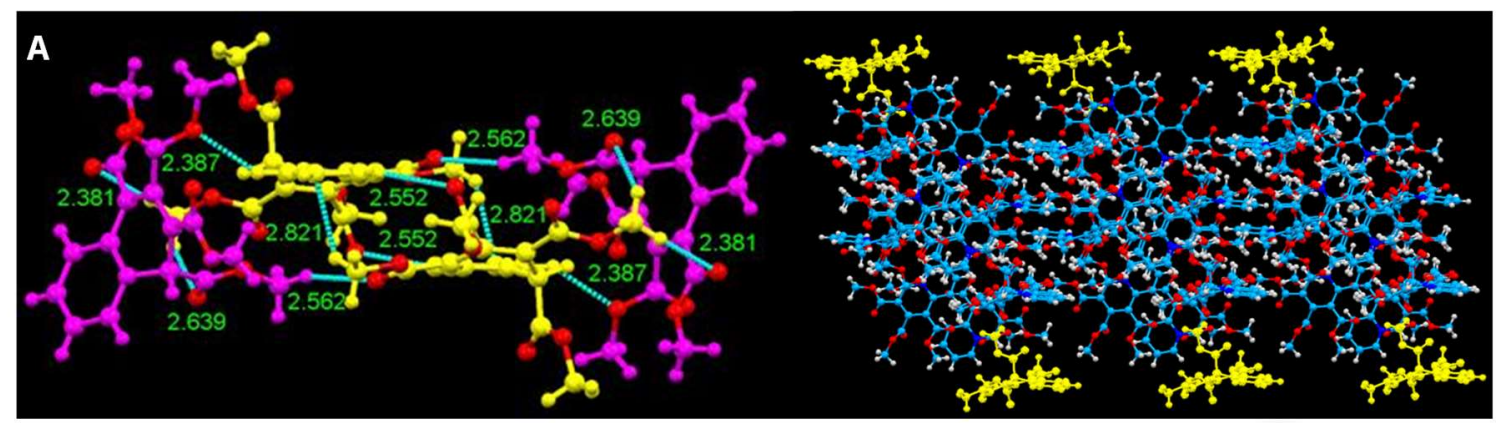

Figure 4. (A) Intermolecular hydrogen bonding interactions (left) and intermolecular packings (right) of QLZ crystal crystallized from DCM/diethyl ether)

\section{Structural Tailoring and Bioapplications}

The above investigations have well demonstrated that QLZ is an ideal RIV-based AIE core, but its luminescence in the aggregate state is not so superior for further application, such as short emission wavelength and no outstanding function, so we further tailored this structure to expand its application. Generally, the construction of typical donor-acceptor (D-A) structure is a good strategy to redshift the emission. ${ }^{[18]}$ Since the quinolizine rings with four electronwithdrawing ester groups can act as an acceptor, we thus considered to introduce the typical electron-donating group, triphenylamine (TPA), to construct a D-A structure. Following this 
idea, we synthesized the target molecule TPA-QLZ (Figure 5A). Similarly, TPA-QLZ was also prepared by one-step simple annulation reaction of $\mathbf{1}$ with $\mathbf{3}$ under mild conditions (see the Supporting Information for details). The structure of TPA-QLZ was characterized using NMR and HRMS with satisfactory results (Figures S10-S12). Compared with QLZ, TPA-QLZ exhibits a longer-wavelength absorption and emission of $475 \mathrm{~nm}$ and $640 \mathrm{~nm}$, respectively (Figure 5B). The AIE feature of TPA-QLZ was further evaluated. With the addition of poor solvent water, the PL intensity of TPA-QLZ was enhanced gradually and reached the maximum at $f_{\mathrm{w}}$ of $90 \%$ (Figure $5 \mathrm{C}$ ). As designed the introduction of TPA moiety indeed could boost the AIE performance, and the $I / I_{0}$ value was 5.8 which is much higher than that of QLZ (Figure 5D). These results indicate that the TPA-QLZ exhibits a much superior AIE characteristic.

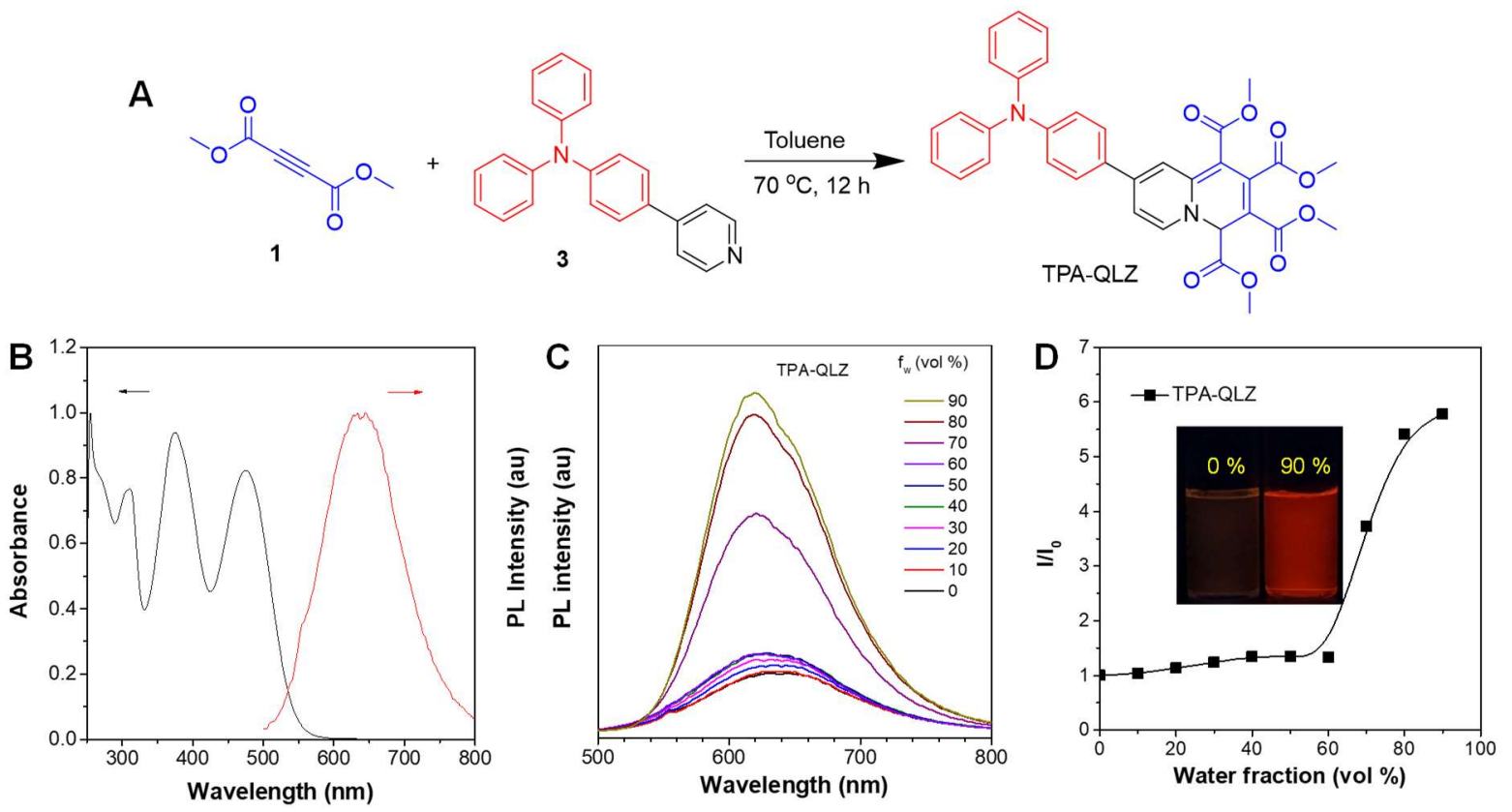

Figure 5. (A) Synthetic route to TPA-QLZ. (B) UV/vis absorption $(C=100 \mu \mathrm{M})$, and PL spectra $\left(\lambda_{\mathrm{ex}}=475 \mathrm{~nm}, 10 \mu \mathrm{M}\right)$ of TPA-QLZ in THF, (C) PL spectra of TPA-QLZ in THF/water mixtures with different water fractions $\left(f_{\mathrm{w}}\right)$. (D) Plot of relative emission intensity $\left(I / I_{0}\right)$ versus the water fraction of the aqueous mixtures of TPA-QLZ $\left(\lambda_{\mathrm{ex}}=475 \mathrm{~nm}, 10 \mu \mathrm{M}\right)$.

It has been proved that the typical D-A structure could facilitate the generation of reactive oxygen species (ROS). ${ }^{[19]}$ So we presumed that TPA-QLZ may have excellent ROS generation 
performance. To confirm this hypothesis, the abilities of total ROS generation were evaluated by using 2,7-dichlorodihydrofluorescein (DCFH-DA) as an indicator. As shown in Figure 6A, in the presence of photosensitizers (PSs) or DCFH-DA alone, the fluorescence intensity changes were negligible even exposed to white light irradiation $\left(24 \mathrm{~mW} \mathrm{~cm}{ }^{-2}\right)$ for $10 \mathrm{~min}$. However, in the presence of TPA-QLZ, the emission intensities of DCFH, which activated by any type of ROS, were rapidly boosted as the irradiation duration increases. After 10 min of irradiation, the fluorescence intensity of DCFH has increased by more than 1200 -fold, which was much superior to the commercially available photosensitizer Chlorin e6 (Ce6, about 400fold enhancement) under the same conditions. It is worth mentioning that the fluorescence intensity of DCFH changed very slightly even in the presence of QLZ, probably due to the lack of D-A structure of QLZ molecules, which is not conducive to ROS generation.

The ROS types were further discriminated by using different ROS probes. ${ }^{[20]}$ We utilized 9,10-anthracenediyl-bis(methylene) dimalonic acid (ABDA) to detect type-II ROS siglet oxygen $\left({ }^{1} \mathrm{O}_{2}\right)$, and utilized hydroxyphenyl fluorescein (HPF) and dihydrorhodamine 123 (DHR123) to test type-I ROS hydroxyl radical $(\bullet \mathrm{OH})$ and superoxide radical $\left(\bullet \mathrm{O}_{2}^{-}\right)$, respectively. As shown in Figure S13A, the absorbance of ABDA decreased significantly with the increasing of irradiation time in the presence of TPA-QLZ, suggesting that it was capable of generating ${ }^{1} \mathrm{O}_{2}$ effectively. Compared with the commercially available Ce6, TPA-QLZ showed comparable ${ }^{1} \mathrm{O}_{2}$ generation capabilities. Moreover, in the presence of TPA-QLZ, the fluorescence intensities of HPF and DHR123 both enhanced obviously with the increasing of irradiation time, implying its strong $\bullet \mathrm{OH}$ and $\bullet \mathrm{O}_{2}{ }^{-}$production abilities (Figure S13B and 13C). By contrast, only slight fluorescence enhancement was observed in the presence of Ce6 under 
the same conditions, further indicating that the TPA-QLZ has excellent $\bullet \mathrm{OH}$ and $\bullet \mathrm{O}_{2}^{-}$ generation capabilities. Taken together, it was reasonable to conclude that the TPA-QLZ can generate both type-II $\left({ }^{1} \mathrm{O}_{2}\right)$ and type-I $\left(\bullet \mathrm{OH}\right.$ and $\left.\bullet \mathrm{O}_{2}{ }^{-}\right)$ROS.

To elucidate the excellent ROS generation of TPA-QLZ, theoretical calculations were performed based on DFT and time-dependent density functional theory (TD-DFT). As demonstrated in Figure S14, the electrons of both the highest occupied molecular orbital (HOMO) and the lowest unoccupied molecular orbital (LUMO) of QLZ are delocalized on the quinolizine rings. However, for TPA-QLZ molecule, the HOMO is predominantly localized on the electron-donating TPA unit, while the LUMO mainly distributes on the electronwithdrawing QLZ core. These results indicate that the construction of D-A structure can facilitate charge separation, thus promoting the occurrence of intersystem crossing (ISC) process from singlet states to triplet states, and then generating ROS by oxidation. ${ }^{[21]}$

The subsequent TD-DFT studies were conducted on both singlet and triplet excited states of QLZ and TPA-QLZ at the B3LYP/6-31G $(\mathrm{d}, \mathrm{p})$ level to explore the nature of ROS generation. As shown in Figure S15, the lowest singlet excited (S1) state can be transferred to triplet states through multiple energy transition channels. The energy gap of $\mathrm{S}_{1}-\mathrm{T}_{1}$ and $\mathrm{S}_{1}-\mathrm{T}_{2}$ of QLZ is 0.565 $\mathrm{eV}$ and $0.446 \mathrm{eV}$, respectively, which are both greater than $0.3 \mathrm{eV}$ and it can be considered that the ISC process is hart to occur. That is why the QLZ molecule cannot produce ROS. However, for TPA-QLZ, the energy gap of $\mathrm{S}_{1}-\mathrm{T}_{2}$ is as low as $0.156 \mathrm{eV}$, indicating that it is prone to ISC process and thus can transfer to triplet state. Besides, the spin-orbit coupling (SOC) constants also prove that the TPA-QLZ molecules are more inclined to transfer to the triplet state through the $\mathrm{S}_{1}-\mathrm{T}_{2}$ pathway. From the above results, we can conclude that the construction of D-A 
structure makes TPA-QLZ molecule more prone to ISC process and thus to produce ROS.

Considering the unique luminescent and ROS generation properties of the obtained TPAQLZ, we further explored its biological applications. For more efficient bioapplications, water suspensions of TPA-QLZ dots were fabricated by nanoprecipitation. Transmission electron microscope results revealed that TPA-QLZ dots exhibited well-dispersible spherical morphology, and the average hydrodynamic diameter of TPA-QLZ dots measured by dynamic light scattering was about $78 \mathrm{~nm}$ (Figure 6B). The cellular uptake and intracellular distribution of TPA-QLZ dots was firstly investigated by confocal laser scanning microscopy (CLSM) imaging. As shown in Figure 6D, TPA-QLZ dots could be largely uptaken by 4T1 cells that emitted bright red fluorescence after incubation for $4 \mathrm{~h}$. Co-localization experiments with MitoTracker Green showed that most of the TPA-QLZ dots accumulated in mitochondria, indicating that TPA-QLZ could selectively label mitochondria and may boost the photodynamic therapy efficiency. ${ }^{[22]}$ In addition, the intracellular ROS generation of TPA-QLZ dots was evaluated by using DCFH-DA as an indicator, which can generate green fluorescence after being oxidized by ROS. As illustrated in Figure 6E, no obvious fluorescence was observed in the control groups (PBS, PBS + light, and TPA-QLZ dots alone), while the group treated with TPA-QLZ dots upon white light irradiation exhibited bright green fluorescence, revealing that TPA-QLZ dots with white light irradiation could efficiently cause intracellular ROS generation.

In light of the great cellular uptake and effective intracellular ROS generation of TPAQLZ dots, the light-induced tumoricidal activities in vitro of TPA-QLZ dots were evaluated through cell counting kit-8 (CCK8) assay. As depicted in Figure 6C and Figure S16, TPA-QLZ 
dots exhibited negligible dark cytotoxicity to $4 \mathrm{~T} 1$ and HeLa tumor cells as well as NIH/3T3 and COS-7 normal cells even at high concentration, validating their excellent biocompatibility. Upon white light irradiation $\left(24 \mathrm{~mW} \mathrm{~cm}^{-2}\right)$ for 5 or $10 \mathrm{~min}$, a dose-dependent phototoxicity against $4 \mathrm{~T} 1$ cells was clearly observed, revealing the significant photodynamic tumoricidal efficacy of TPA-QLZ dots. Furthermore, live/dead staining experiments by using fluorescein diacetate (FDA, green color for live cells)/propidium iodide (PI, red color for dead cells) were assessed to reveal the photodynamic tumoricidal efficacy of TPA-QLZ dots intuitively. As displayed in Figure 6F, consistent with the CCK-8 assay results, 4T1 cells treated with TPAQLZ dots plus white light irradiation exhibited bright red fluorescence and presented death state, but strong green fluorescent signal was observed in the PBS, PBS + light, and TPA-QLZ dots alone groups, indicating that TPA-QLZ dots have efficient and controllable photoablation capabilities. Taken together, these results definitely indicate that TPA-QLZ is significantly powerful to ablate cancer cells by virtue of PDT treatment. 

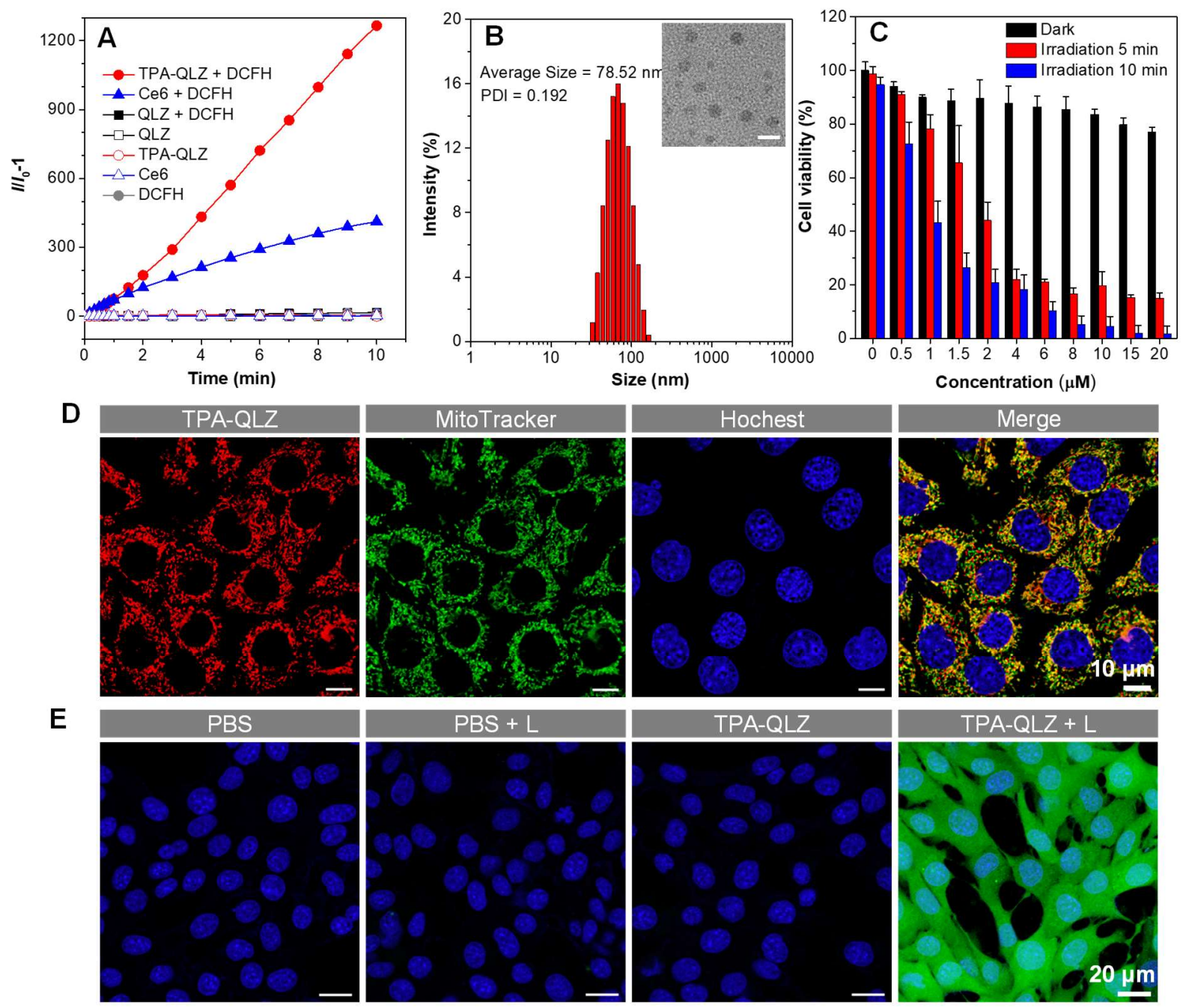

$\mathbf{F}$
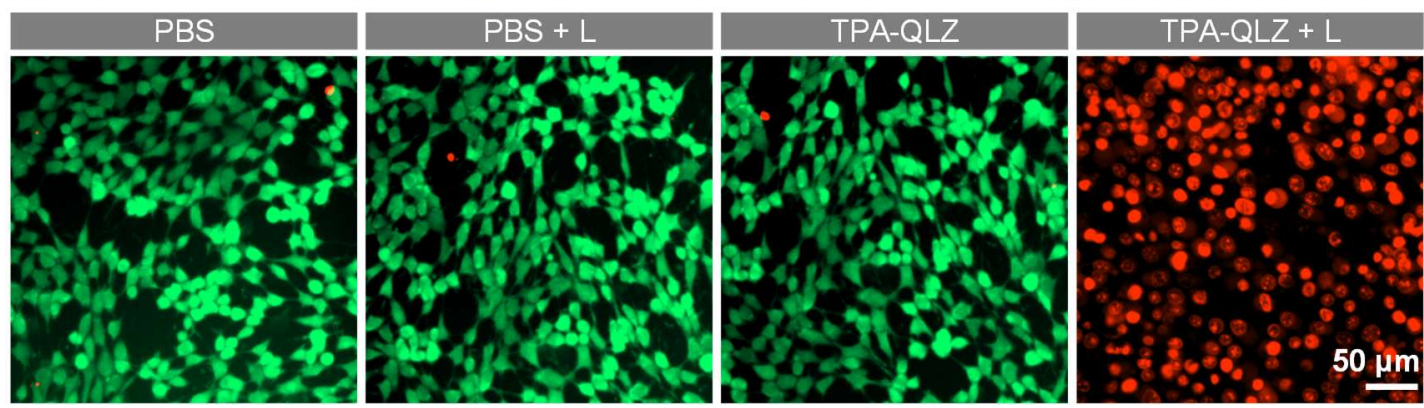

Figure 6. (A) ROS generation profiles of photosensitizers $(2 \mu \mathrm{M})$ by using DCFH-DA (for overall ROS detection) as indicator upon white light irradiation $\left(24 \mathrm{~mW} \mathrm{~cm}^{-2}\right)$. (B) DLS size distribution and TEM image (inset) of TPA-QLZ dots in aqueous solution. Scale bar: $100 \mathrm{~nm}$. (C) Relative viabilities of 4T1 cells after treated with TPA-QLZ dots at various concentrations in the dark or after white light irradiation for 5 or 10 min determined by CCK-8 assay. (D) CLSM imaging of 4T1 cells after co-staining with TPA-QLZ dots, MitoTracker Green and Hoechst 33342. (E) Intracellular ROS generation of 4T1 cells after different treatments indicated by DCFH-DA. (F) Live/dead cell staining of 4T1 cells after different treatments.

Inspired by the excellent photodynamic therapeutic efficacy in vitro, TPA-QLZ was 
further estimated for in vivo applications on the 4T1-tumor-bearing BALB/c nude mice. After intratumor injection of TPA-QLZ dots, a bright fluorescence signal was detected at the tumor site after $1 \mathrm{~h}$ injection (Figure 7A). With the extension of observation time, the fluorescence signals of tumor region declined gradually as a consequence of the metabolism of TPA-QLZ dots. Subsequently, the in vivo phototherapeutic study was performed. After $1 \mathrm{~h}$ of postinjection of TPA-QLZ dots or saline, the tumor regions of the mice were exposed to white light irradiation $\left(0.1 \mathrm{~W} \mathrm{~cm}^{-2}\right)$ for $20 \mathrm{~min}$ at the predetermined groups. As depicted in Figure $7 \mathrm{~B}$, the tumor volumes increased sharply to almost the same degree in control groups during the study period, confirming that light irradiation alone or TPA-QLZ dots administration alone had no suppression effect on tumor growth. Excitingly, upon the introduction of white light irradiation, TPA-QLZ dots revealed remarkable tumor growth inhibition efficiency, and the inhibition rate was as high as $73.6 \%$ (Figure 7B and Figure S17). In order to further inspect the in vivo PDT efficacy of the therapeutic agent, the histological and immunohistochemical evaluation of the dissected tumors was conducted. As shown in Figure 7C, compared to the control groups, lightinduced ROS based on TPA-QLZ dots could lead to the most severe apoptosis or necrosis of tumor cells (see panels H\&E and TUNEL), and Ki67-positive proliferating tumor cells as well as the new microvessels were rarely found in this phototherapeutic group (see panels Ki67 and CD31). These results convincingly demonstrate that the TPA-QLZ can effectively induce tumor cell apoptosis as well as inhibit tumor cell proliferation and tumor vessel generation by fluorescence imaging-guided PDT pathway. ${ }^{[23]}$

Considering the biological safety of theranostic agent is important in clinic, the in vivo biosecurity of TPA-QLZ dots was carefully estimated. Preliminarily, no significant body 
weight loss was found for all the treated mice during the treatment course (Figure 7D). In addition, after intravenous injection of TPA-QLZ dots for 7 days, the normal mice were sacrificed and the blood was collected for hematology and blood biochemical analyses. As displayed in Figure S18 and Table S2 (Supporting Information), the blood biochemistry parameters of hepatic function markers (ALB, ALT and AST) and renal function markers (BUN, CREA and UA), as well as the routine blood indexes in the TPA-QLZ-administered group were also normal as saline-administered group, revealing negligible systemic side effects of TPAQLZ dots. Moreover, the main organs (heart, liver, spleen, lung, and kidney) of the mice were also exfoliated for histological analysis (Figure S19). As shown in H\&E-stained slices, no noticeable organ damage or inflammatory lesions were found in the major organs after treatment with TPA-QLZ dots, offering another solid evidence for TPA-QLZ dots with excellent biocompatibility and biosafety. 
A
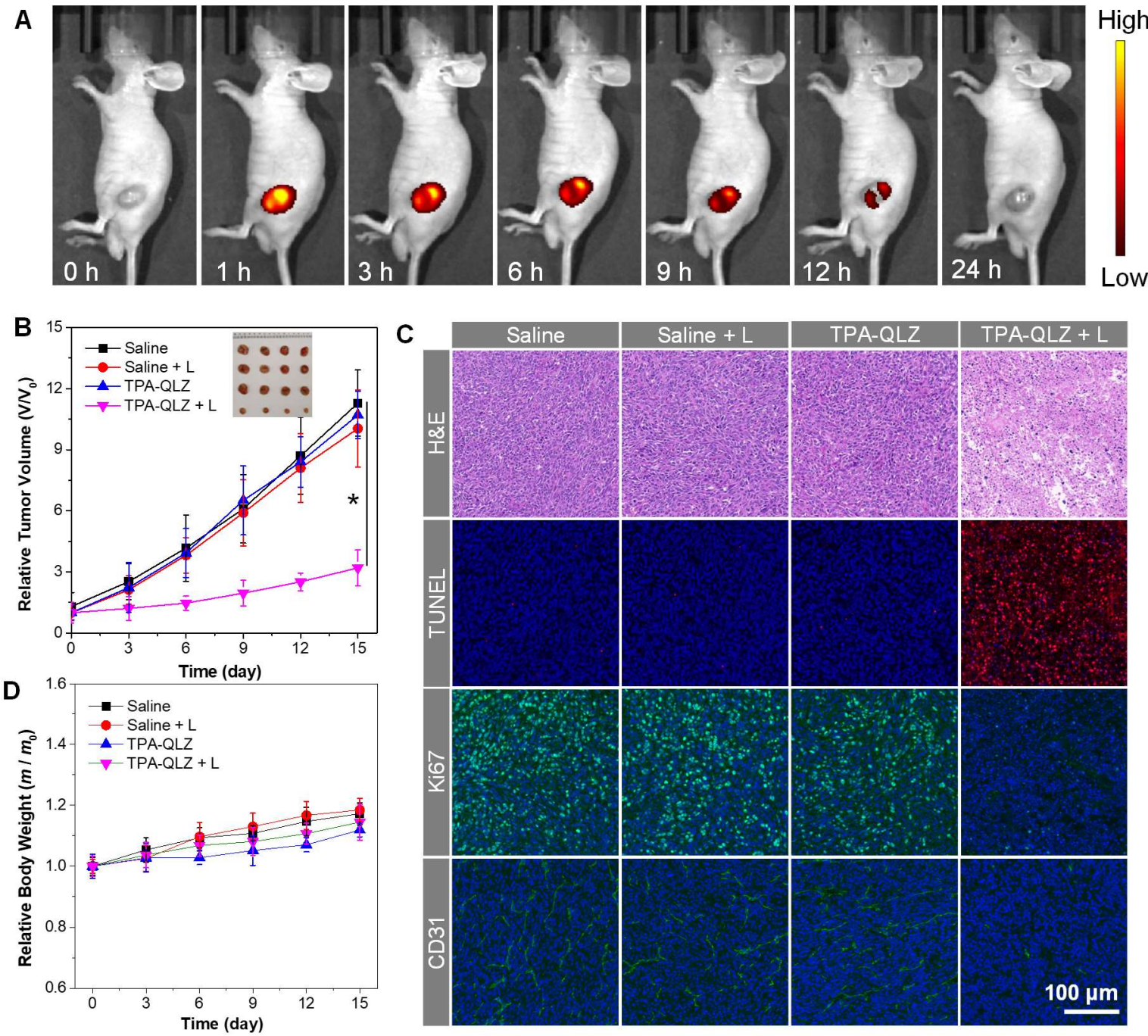

Figure 7. In vivo fluorescence-imaging-guided PDT effect of TPA-QLZ dots on 4T1-tumorbearing mice. (A) Time-lapse fluorescence images of mice after administration of TPA-QLZ dots. (B) Tumor growth curves and (D) body weight changes of tumor-bearing mice with various treatments $(* p<0.05)$. Inset: Digital photos of the tumors harvested from different treatment groups on day 15. (C) Histological H\&E, TUNEL (red fluorescence), Ki67 (green fluorescence), and CD31 (green fluorescence) staining analysis of tumor tissues after different treatments.

\section{Conclusion}

In summary, we have constructed a novel quinolizine AIE system based on RIV mechanism. The QLZ molecule contains a unique non-planar structure, which can undergo a bent-to-planar vibration upon excitation. Additionally, it has been demonstrated that the crystal of QLZ exhibited dual emission corresponding to two different molecular motion states, so it 
could act as an ideal model to visualize single-molecule motion and macroscopic molecular motion via fluorescence change. Furthermore, the QLZ structure was elaborately tailored and a D-A type of TPA-QLZ molecule with much superior AIE and ROS generating properties was constructed, which can generate both type-II $\left({ }^{1} \mathrm{O}_{2}\right)$ and type-I $\left(\bullet \mathrm{OH}\right.$ and $\left.\bullet \mathrm{O}_{2}{ }^{-}\right)$ROS under white light irradiation. Accordingly, TPA-QLZ can realize highly efficient fluorescence imagingguided PDT towards cancer. Therefore, this work not only provides an ideal model to visualize complicated molecular motions via constructing a novel AIE core, but also demonstrates the great application potential of new AIE system.

\section{Acknowledgements}

We are grateful for financial support from the National Natural Science Foundation of China (22101028 and 21788102), the start-up funding from Beijing Normal University (310432102 and 310432103), the Innovation and Technology Commission (ITCCNERC14SC01), the Science and Technology Plan of Shenzhen (JCYJ20160229205601482, JCYJ20180507183832744 and JCYJ20170818113602462), and the Research Grants Council of Hong Kong (16305618, 16304819, 16305320, N-HKUST609/19, C6009-17G, and C601420W/16).

\section{Conflict of interest}

The authors declare no conflict of interest.

\section{References}

[1] a) S. Erbas-Cakmak, D. A. Leigh, C. T. McTernan, A. L. Nussbaumer, Chem. Rev. 2015, 115, 10081-10206; b) P. Alam, N. L. C. Leung, Y. Cheng, H. Zhang, J. Liu, W. Wu, R. T. K. Kwok, J. W. Y. Lam, H. H. Y. Sung, I. D. Williams, B. Z. Tang, Angew. Chem. Int. Ed. 2019, 58, 4536-4540; c) D. Roke, S. J. Wezenberg, B. L. Feringa, Proc. Natl. Acad. Sci. U. S. A. 2018, 115, 9423-9431. 
[2] a) E. H. G. Backus, A. Eichler, A. W. Kleyn, M. Bonn, Science 2005, 310, 1790-1793; b) J. Zhang, B. He, W. Wu, P. Alam, H. Zhang, J. Gong, F. Song, Z. Wang, H. H. Y. Sung, I. D. Williams, Z. Wang, J. W. Y. Lam, B. Z. Tang, J. Am. Chem. Soc. 2020, 142, 1460814618.

[3] a) S. Liu, Y. Li, H. Zhang, Z. Zhao, X. Lu, J. W. Y. Lam, B. Z. Tang, ACS Mater. Lett. 2019, 1, 425-431; b) H. Wang, Q. Li, J. Zhang, H. Zhang, Y. Shu, Z. Zhao, W. Jiang, L. Du, D. L. Phillips, J. W. Y. Lam, H. H. Y. Sung, I. D. Williams, R. Lu, B. Z. Tang, J. Am. Chem. Soc. 2021, 143, 9468-9477; c) D. Tu, J. Zhang, Y. Zhang, H. H. Y. Sung, L. Liu, R. T. K. Kwok, J. W. Y. Lam, I. D. Williams, H. Yan, B. Z. Tang, J. Am. Chem. Soc. 2021, $143,11820-11827$.

[4] a) N. L. C. Leung, N. Xie, W. Yuan, Y. Liu, Q. Wu, Q. Peng, Q. Miao, J. W. Y. Lam, B. Z. Tang, Chem. Eur. J. 2014, 20, 15349-15353; b) Z. Zhao, H. Zhang, J. W. Y. Lam, B. Z. Tang, Angew. Chem. Int. Ed. 2020, 59, 9888-9907; c) Q. Peng, Z. Shuai, Aggregate 2021, 2, e91; d) S. Ma, S. Du, G. Pan, S. Dai, B. Xu, W. Tian, Aggregate 2021, 2, e96.

[5] a) J. Zhang, B. He, Y. Hu, P. Alam, H. Zhang, J. W. Y. Lam, B. Z. Tang, Adv. Mater. 2021, 33, 2008071; b) B. He, J. Zhang, J. Zhang, H. Zhang, X. Wu, X. Chen, K. H. S. Kei, A. Qin, H. H. Y. Sung, J. W. Y. Lam, B. Z. Tang, Adv. Sci. 2021, 8, 2004299; c) J. Zhang, Q. Liu, W. Wu, J. Peng, H. Zhang, F. Song, B. He, X. Wang, H. H. Y. Sung, M. Chen, B. S. Li, S. H. Liu, J. W. Y. Lam, B. Z. Tang, ACS Nano 2019, 13, 3618-3628; d) J. Qi, H. Ou, Q. Liu, D. Ding, Aggregate 2021, 2, 95-113; e) P. Alam, N. L. C. Leung, J. Zhang, R. T. K. Kwok, J. W. Y. Lam, B. Z. Tang, Coord. Chem. Rev. 2021, 429, 213693; f) M. Kang, Z. Zhang, N. Song, M. Li, P. Sun, X. Chen, D. Wang, B. Z. Tang, Aggregate 2020, 1, 80106.

[6] J. Mei, Y. Hong, J. W. Y. Lam, A. Qin, Y. Tang, B. Z. Tang, Adv. Mater. 2014, 26, 54295479.

[7] Z. Zhao, X. Zheng, L. Du, Y. Xiong, W. He, X. Gao, C. Li, Y. Liu, B. Xu, J. Zhang, F. Song, Y. Yu, X. Zhao, Y. Cai, X. He, R. T. K. Kwok, J. W. Y. Lam, X. Huang, D. Lee Phillips, H. Wang, B. Z. Tang, Nat. Commun. 2019, 10, 2952.

[8] a) A. Schwartz, Z. Pál, L. Szabó, K. Simon, I. Hermecz, Z. Mészáros, J. Heterocycl. Chem. 1987, 24, 645-650; b) T. R. Govindachari, B. S. Thyagarajan, Proc. Indian Acad. Sci. 1954, 
39, 232-239.

[9] O. Diels, K. Alder, Justus Liebigs Ann. Chem. 1932, 498, 16-49.

[10] a) R. Gujjarappa, N. Vodnala, C. C. Malakar, ChemistrySelect 2020, 5, 8745-8758; b) A. R. Sherman, J. Genovino, Pyridine, in Encyclopedia of Reagents for Organic Synthesis, 2015, pp. 1-8; c) S. Shimizu, N. Watanabe, T. Kataoka, T. Shoji, N. Abe, S. Morishita, H. Ichimura, Pyridine and Pyridine Derivatives, in Ullmann's Encyclopedia of Industrial Chemistry yridine and Pyridine Derivatives, 2000.

[11] a) J. L. Archibald, D. R. Beardsley, T. J. Ward, J. F. Waterfall, J. F. White, J. Med. Chem. 1983, 26, 416-420; b) I. J. Kim, Y. J. Park, J. il Kim, K. T. Lee, S. K. Kim, Arch. Pharmacal Res. 1997, 20, 476-479; c) Y. Liu, Y. Cui, L. Lu, Y. Gong, W. Han, G. Piao, Arch. Pharm. 2020, 353, 2000120.

[12] Z. Zhang, Y.-S. Wu, K.-C. Tang, C.-L. Chen, J.-W. Ho, J. Su, H. Tian, P.-T. Chou, J. Am. Chem. Soc. 2015, 137, 8509-8520.

[13] H. Zhang, Z. Zhao, A. T. Turley, L. Wang, P. R. McGonigal, Y. Tu, Y. Li, Z. Wang, R. T. K. Kwok, J. W. Y. Lam, B. Z. Tang, Adv. Mater. 2020, 32, 2001457.

[14] a) J. Yang, M. Fang, Z. Li, Aggregate 2020, 1, 6-18; b) J. Mei, N. L. C. Leung, R. T. K. Kwok, J. W. Y. Lam, B. Z. Tang, Chem. Rev. 2015, 115, 11718-11940; c) J. Yang, Z. Chi, W. Zhu, B. Z. Tang, Z. Li, Sci. China: Chem. 2019, 62, 1090-1098.

[15] a) Z. Shuai, Q. Peng, Natl. Sci. Rev. 2017, 4, 224-239; b) H. Zhang, J. Liu, L. Du, C. Ma, N. L. C. Leung, Y. Niu, A. Qin, J. Sun, Q. Peng, H. H. Y. Sung, I. D. Williams, R. T. K. Kwok, J. W. Y. Lam, K. S. Wong, D. L. Phillips, B. Z. Tang, Mater. Chem. Front. 2019, 3, 1143-1150.

[16] J. Zhang, L. Hu, K. Zhang, J. Liu, X. Li, H. Wang, Z. Wang, H. H. Y. Sung, I. D. Williams, Z. Zeng, J. W. Y. Lam, H. Zhang, B. Z. Tang, J. Am. Chem. Soc. 2021, 143, 9565-9574.

[17] D. Su, C. L. Teoh, L. Wang, X. Liu, Y.-T. Chang, Chem. Soc. Rev. 2017, 46, 4833-4844.

[18] J. Zhang, B. He, Y. Hu, P. Alam, H. Zhang, J. W. Y. Lam, B. Z. Tang, Adv. Mater. 2021, 33, 2008071.

[19] a) Q. Li, Y. Li, T. Min, J. Gong, L. Du, D. L. Phillips, J. Liu, J. W. Y. Lam, H. H. Y. Sung, I. D. Williams, R. T. K. Kwok, C. L. Ho, K. Li, J. Wang, B. Z. Tang, Angew. Chem. Int. Ed. 2020, 59, 9470-9477; b) S. Xu, Y. Yuan, X. Cai, C.-J. Zhang, F. Hu, J. Liang, G. Zhang, 
D. Zhang, B. Liu, Chem. Sci. 2015, 6, 5824-5830.

[20] a) Y. Nosaka, A. Y. Nosaka, Chem. Rev. 2017, 117, 11302-11336; b) A. Gomes, E. Fernandes, J. L. F. C. Lima, J. Biochem. Bioph. Methods 2005, 65, 45-80.

[21] C. Chen, X. Ni, H.-W. Tian, Q. Liu, D.-S. Guo, D. Ding, Angew. Chem. Int. Ed. 2020, 59, 10008-10012.

[22] L. Yang, P. Gao, Y. Huang, X. Lu, Q. Chang, W. Pan, N. Li, B. Tang, Chin. Chem. Lett. 2019, 30, 1293-1296.

[23] X. Ren, S. Zhang, L. Liu, B. Xu, W. Tian, Nanotechnology 2021, 32, 502008. 